\title{
Análise de Funções de Similaridade para Verificação do Conteúdo de Mensagens em Fóruns de Discussão
}

\author{
Danielle C. Medeiros ${ }^{1}$, José Eustáquio Rangel de Queiroz ${ }^{1}$, Joseana M. F. R. \\ Araújo ${ }^{1}$ \\ ${ }^{1}$ Departamento de Sistemas e Computação \\ Universidade Federal de Campina Grande (UFCG) - Campina Grande, PB - Brasil \\ daniellemedeiros@copin.ufcg.edu.br,\{rangel,joseana\}@dsc.ufcg.edu.br
}

\begin{abstract}
In distance learning courses, evaluating the effectiveness and the efficiency of learning activities, such as students' participation throughout the courses, can be time consuming and susceptible to the strong influence of human subjectivity. In such a task, AI techniques can be useful to assist the teacher. A pilot study of similarity functions applied to this context is presented in this article, in order to determine its applicability for the evaluation of messages in discussion forums. Although there is a need for further studies to determine evaluation indicators, it was statistically validated the effectiveness and efficiency of the use of similarity measures.
\end{abstract}

Resumo. Em cursos de EAD, avaliar a eficácia e a eficiência das atividades de aprendizagem, tal como a participação dos alunos ao longo do curso, pode ser uma tarefa onerosa e passivel de forte influência da subjetividade humana. Técnicas de IA podem ser úteis para auxiliar o professor na realização de tarefas dessa natureza. Neste artigo, é apresentado um estudo da aplicação de funções de similaridade ao contexto de EaD, com o propósito de analisar sua aplicabilidade ao apoio à avaliação de mensagens em fóruns de discussão. Embora exista a necessidade de estudos mais aprofundados sobre a definição de indicadores de avaliação aplicáveis a esse contexto, a eficácia e a eficiência da aplicação de medidas de similaridade foi validada estatisticamente.

\section{Introdução}

No Brasil, há diversos estudos com foco na avaliação da aprendizagem nas diferentes modalidades de ensino. Embora a quantidade de pesquisas neste sentido tenha aumentado, ainda há certa escassez de estudos, tanto empíricos, quanto teóricos, no âmbito da Educação a Distância (EaD) [Leite et al. 2011]. Em EaD, quando não é possível o contato presencial entre professor e alunos, os meios para realizar a avaliação do aluno, em especial sua participação ao longo do curso, encontram-se em funcionalidades disponíveis em Ambientes Virtuais de Aprendizagem (AVA). O Moodle [Dougiamas 2001] e o Blackboard [Blackboard Inc. 1997] são exemplos de AVA amplamente utilizados em cursos online. 
Buscando prover a interação entre os alunos, essas ferramentas disponibilizam algumas funcionalidades, tais como fóruns de discussão assíncronos, que possibilitam, a partir de um tema determinado pelo professor, a interação entre os alunos, que compartilham ideias e conhecimentos. Os participantes do fórum têm acesso ao histórico das mensagens postadas e recebidas, podendo revisá-las, caso a mensagem seja de sua autoria, acrescentando comentários às mensagens postadas pelos colegas. Assim, esta funcionalidade tornou-se um elemento importante para promover $\mathrm{o}$ compartilhamento de experiências, tão importante em cursos não presenciais [Calvani et al. 2009].

No entanto, um dos obstáculos impostos pelo uso de fóruns de discussão como forma de avaliação da participação dos alunos é o grande dispêndio de tempo por parte do professor, dependendo do fluxo de mensagens presentes no fórum. Muitas vezes, há um grande número de mensagens a serem avaliadas pelo docente [Andresen 2009], o que pode tornar tal atividade impraticável.

Em sua maioria, os AVA comumente utilizados oferecem informações estatísticas, tais como a quantidade de mensagens que um determinado discente enviou ao fórum ou o número de comentários deixados em resposta a mensagens de colegas. A utilização deste tipo de informação, em geral, não é suficiente para avaliar o nível de atividade dos alunos, já que os dados estatísticos acabam levando em consideração apenas a quantidade e não a qualidade das postagens [Erlin et al. 2009]. Uma forma de minimizar este problema consiste na adoção de técnicas de automatização das formas de avaliação, visando a fornecer meios para auxiliar o professor no monitoramento das atividades dos alunos e nos processos decisórios, ao avaliar a participação de cada aluno.

Para automatizar atividades de avaliação em cursos online, faz-se necessária, inicialmente, a realização de um estudo dos indicadores comumente utilizados pelos professores. Hrastinski (2008) sugere como indícios de atividade participativa dos alunos a utilização do tempo de acesso ao ambiente virtual, da frequência de leitura de postagens e da quantidade de mensagens postadas. Andresen (2009) menciona alguns estudos que tratam do sucesso e efetividade da adoção desses indicadores na avaliação da participação em cursos online. No entanto, esses indicadores não refletem a qualidade das informações discutidas nem fornecem meios para verificar se as postagens se mantêm no âmbito do assunto proposto.

Um dos grandes obstáculos para a verificação automática do conteúdo em fóruns é a subjetividade associada à avaliação realizada pelo professor. Convencionalmente, o docente é capaz de verificar, por exemplo, se o conteúdo das mensagens é pertinente ao tópico em discussão, se as informações trazidas apresentam argumentos originais ou se seu conteúdo foi extraído integralmente da web. Neste contexto, o uso de técnicas de Inteligência Artificial (IA) pode constituir-se uma ferramenta adequada para a resolução de tais problemas.

No âmbito da Inteligência Artificial, existem várias estratégias para a representação do conhecimento. Uma das técnicas aplicáveis à verificação textual é o cálculo do grau de similaridade entre termos, que possibilita verificar se há relação entre dois conjuntos de dados. Dentre as funções de similaridade mais populares estão: a 
Similaridade dos Cossenos, a Distância Euclidiana, o Índice de Similaridade de Jaccard e os algoritmos Dice's Coefficient e Overlap [Tan et al. 2005].

No presente artigo, apresenta-se, neste sentido, um estudo piloto sobre o uso de funções de similaridade como indicador para a verificação da participação dos alunos em fóruns assíncronos de EaD. Para tanto, foram utilizadas mensagens extraídas de um curso online de Computação Gráfica. Cada mensagem postada foi avaliada por um professor especialista, recebendo um valor entre 0 (zero) e 1 (um). O professor descreveu, ainda, um conjunto de palavras-chave pertinentes ao assunto discutido no fórum, o qual foi utilizado como indicador de participação a ser estudado. Assim, foi efetuado o cálculo da similaridade entre o conjunto de palavras previamente definido pelo docente e cada mensagem do fórum, utilizando-se 4 (quatro) algoritmos: Similaridade dos Cossenos, Distância Euclidiana, Índice de Coeficiente de Jaccard e Dice's Coefficient. O objetivo do estudo foi determinar a existência de diferenças estatísticas entre as funções de similaridade estudadas e, por extensão, a verificação daquela que produzia os melhores resultados. Para examinar a eficiência das funções de similaridade, cada valor obtido a partir de uma função foi confrontado com os valores reais atribuídos pelo especialista.

Dentre as funções analisadas, foi possível verificar que os melhores resultados foram obtidos com a utilização da função de Similaridade dos Cossenos. Além disto, a partir da observação do conteúdo das mensagens e do comportamento dos dados simulados, concluiu-se, também, que utilizar unicamente um indicador não é suficiente para avaliar a participação do aluno, uma vez que este não captura a subjetividade aplicada na avaliação dos discentes.

\section{Revisão de Literatura}

A utilização de métodos para analisar mensagens em fóruns, com o objetivo de verificar a participação de alunos em cursos online, têm sido um dos grandes desafios para professores desta modalidade de ensino. Lin et al. (2009) apresentaram uma forma de definir tópicos a partir do conteúdo de fóruns de discussão, fazendo uso de técnicas de mineração de dados. Para isso, eles propuseram a classificação das informações enviadas ao fórum (e.g., anúncio, afirmação, pergunta, explicação, etc.). $\mathrm{O}$ foco do estudo era a verificação da eficiência do uso de mineração de dados para tal fim. Assim, os resultados obtidos foram confrontados com a análise subjetiva realizada pelo professor. Para os autores, a presença do professor ao longo da discussão pode contribuir positivamente para a evolução da aprendizagem dos alunos.

No estudo realizado por Júnior e Esmin (2012), o ambiente proposto em [Junior et al. 2011], para realização do monitoramento de fóruns, foi reestruturado, com a utilização de um algoritmo de aprendizado semi-supervisionado. No estudo anterior, o ambiente classificava as mensagens em Positivo ou Negativo. As mensagens que recebiam o classificador Negativo continham dúvidas, conteúdo indevido ou insatisfação. Todas as demais mensagens eram classificadas como Positivo. Na pesquisa atual, os autores restringiram a classificação das mensagens em duas categorias Dúvidas e Esclarecimentos a Dúvidas. Para isso, eles utilizaram um vetor de características, em que cada palavra de uma mensagem constitui uma dimensão deste vetor. As mensagens também passaram por um tratamento para remoção de palavras não relevantes para a diferenciação entre documentos (e.g., artigos, preposições, etc.) e algumas delas foram 
classificadas manualmente. Ao final, apesar dos bons resultados obtidos, eles concluem que a criação de ambientes similares ao desenvolvido, em muitos casos, pode ser complexa.

Romero et al. (2013) sugeriram a utilização de um módulo para o Moodle para prever a aprovação ou reprovação dos alunos em determinada disciplina, a partir de sua participação em fóruns de discussão. Para isso, eles fizeram uso de técnicas de mineração de dados e de indicadores de participação quantitativos, qualitativos e sociais. Alguns dos indicadores contemplavam dados como a quantidade de palavras e o total de sentenças contidas em cada mensagem, além da média de avaliação das mensagens fornecida pelo próprio professor. Para realizar a previsão, eles fizeram uso de dois métodos de mineração: classificação e clustering. Os autores verificaram que as mensagens que possuíam conteúdo pertinente ao tema principal do fórum demonstraram maior eficiência no contexto de seu estudo. Como uma de suas conclusões, os autores apontaram como os principais indicadores para verificação do uso do fórum: a verificação de palavras, a avaliação das mensagens, a quantidade de mensagens enviadas e os indicadores sociais. Ao final, eles afirmaram que seria útil o uso de uma abordagem para avaliação automática das mensagens, uma vez que o processo manual é monótono e exige um tempo considerável.

Elia e Chamovitz (2009) apresentaram uma ferramenta de apoio aos estudos sobre a avaliação de mensagens em fóruns de discussão. A ferramenta CQMsg foi produto de pesquisas sobre fóruns categorizados e seu objetivo era a criação de um protocolo de avaliação, adaptável às necessidades de cada avaliador. Esta ferramenta classificava as mensagens em categorias, de acordo com uma taxonomia de classificação definida pelo criador do fórum. Para possibilitar que a ferramenta fosse utilizada de forma mais conveniente, os alunos foram incentivados a construir, ao início de suas mensagens, sentenças que refletissem o seu conteúdo. Assim, os avaliadores poderiam avaliar as mensagens, seguindo um conjunto de elementos definidos previamente pelo professor. No entanto, a análise das avaliações das mensagens foi feita de forma puramente estatística. Os autores afirmaram, ao final, que estão investigando o uso de técnicas de IA, como a lógica nebulosa, para este fim.

De acordo com a revisão bibliográfica efetuada, pôde-se constatar a adoção de indicadores, dentre eles a verificação do conteúdo de mensagens de fóruns, como forma de avaliar a participação do aluno. Observou-se, também, que, apesar de fornecer meios para organizar e facilitar esta avaliação, ainda é de inteira responsabilidade de um supervisor humano, a triagem e análise dos dados fornecidos, utilizando-os como apoio a sua avaliação final. É possível verificar, portanto, a necessidade de se estudar uma forma de avaliar as mensagens automaticamente, visando a facilitar a atividade de análise das mensagens realizada pelo professor.

\section{Abordagem Proposta}

No estudo-piloto realizado, pretendeu-se analisar, dentre as funções de similaridade mais utilizadas para mineração de dados, qual, se aplicável, seria a mais adequada para avaliar mensagens de fóruns em cursos de EaD. Para tanto, foi realizado um experimento, seguido de um processamento estatístico dos dados coletados via experimentação. 
A pesquisa teve como objetivo validar o uso de funções de similaridade para a determinação automática do nível de participação de alunos em cursos de EaD, utilizando mensagens trocadas por alunos em fóruns de discussão. Assim sendo, foram utilizadas as mensagens de um fórum de discussão, em um curso de $\mathrm{EaD}$ de curta duração, da disciplina de Computação Gráfica. Um indicador de participação estudado consistiu na similaridade entre as palavras-chave (termos comuns e relevantes para $o$ tema discutido no fórum) definidas pelo professor especialista e as mensagens enviadas pelo aluno. O estudo realizado pode ser visualizado a partir do diagrama em blocos da Figura 1, descrita mais detalhadamente na seção 3.1.

Figura 1. Diagrama em blocos da abordagem proposta.

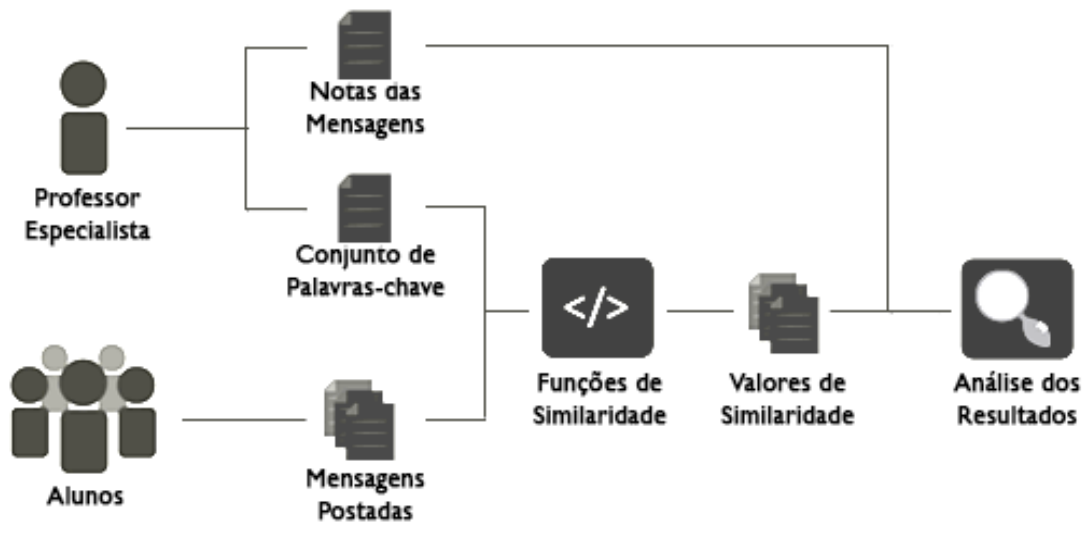

\subsection{Estudo Empírico}

A amostra utilizada para a análise foi obtida de fóruns de discussão de um ambiente virtual de ensino de um curso online de Computação Gráfica, consistindo de pares de mensagens e sua respectiva avaliação por um professor especialista.

Para este estudo, o professor especialista definiu também um conjunto de palavras-chave que julgou relevantes para a discussão e para a atribuição do nível de participação do aluno (exemplos são ilustrados na Tabela 1). Observa-se, a partir da tabela, que várias palavras-chave apresentam sufixos semelhantes. Portanto, foi realizado um tratamento anterior à inserção destas palavras na base de conhecimento, de forma que apenas os sufixos foram utilizados, o que reduziu consideravelmente o conjunto de palavras definido pelo especialista.

Para a realização do experimento, considerou-se a atribuição de nota:

- Máxima, 1 (um), às mensagens contendo todas as palavras-chave descritas pelo professor;

- Mínima, 0 (zero), às mensagens sem nenhuma das palavras-chave descritas pelo professor;

- Intermediária, entre 0 (zero) e 1 (um), às mensagens contendo algumas das palavras-chave.

Tabela 1. Exemplo de algumas palavras-chave do conjunto definido pelo professor especialista, de acordo com o tema definido para o fórum. 


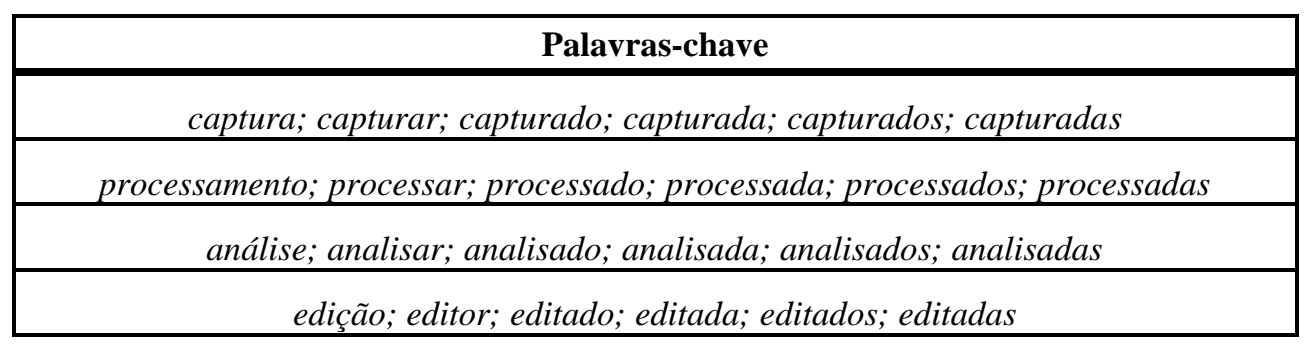

As medidas de similaridade investigadas foram: Similaridade dos Cossenos, Distância Euclidiana, Índice de Coeficiente de Jaccard e Dice's Coefficient [Tan et al. 2005].

Para cada função de similaridade, foi gerado um conjunto de valores entre 0 (zero) e 1 (um), representando o valor da similaridade de cada mensagem em relação ao conjunto de palavras-chave determinado pelo especialista.

Assim, em função do objetivo do estudo foram formulados alguns questionamentos, apresentados a seguir, com o intuito de proporcionar melhor entendimento do que se pretendia investigar.

P1. Há diferença estatística entre os valores simulados obtidos pelas funções de similaridade estudadas, quando confrontados com os valores reais determinados pelo especialista?

P2. Na existência de diferenças entre os valores observados, qual das funções estudadas apresenta resultados mais próximos aos valores reais?

Para responder tais contestações, foi realizada uma análise estatística. Os dados presentes na amostra utilizada para o experimento foram compostos por 37 mensagens extraídas de fóruns de discussão assíncronos, disponibilizados em um curso de Computação Gráfica ministrado no Moodle, de uma universidade particular do estado da Paraíba - Brasil, durante um período de um mês.

Os dados estudados para verificação estatística consistiram em: uma variável dependente do tipo quantitativa contínua, contida no intervalo $[0,1]$, que determinava o valor da similaridade entre a mensagem e o conjunto de palavras-chave definido pelo especialista (indicador adotado); e uma variável independente do tipo qualitativa nominal, que representava a função de similaridade.

Como o experimento realizado utilizou os resultados gerados a partir da aplicação de funções matemáticas determinísticas, não houve necessidade de repetição de experimentos. O fator único estudado foi o Valor de Similaridade. Este fator possuiu quatro níveis categóricos: Função de Similaridade dos Cossenos, Função de Distância Euclidiana, Função da Similaridade de Jaccard e Função Dice's Coeffient [Tan et al. 2005].

Para apoiar o experimento, foram utilizadas as seguintes ferramentas computacionais: ambiente de desenvolvimento integrado Eclipse [Foss 2012], para auxiliar o desenvolvimento do experimento, e o software R Statistics (Ihaka e Gentleman 1993], para realização da análise estatística.

O experimento foi dividido em 3 (três) etapas, a saber: (i) tratamento da amostra; (ii) execução dos algoritmos de similaridade; e (iii) análise dos resultados. $\mathrm{Na}$ primeira etapa da experimentação, foi realizada uma análise dos dados da amostra e o 
tratamento automático do texto, consistindo na retirada de pontuações e acentuações gráficas e na redução de palavras com mesmo sufixo. A segunda etapa consistiu na execução dos algoritmos de similaridade listados anteriormente para o conjunto de mensagens da amostra, resultando na coleta de quatro conjuntos de valores de similaridade, um para cada algoritmo executado. A terceira e última etapa consistiu na análise estatística dos dados simulados. Após uma análise descritiva inicial, verificou-se que o comportamento de alguns grupos de dados não seguia uma distribuição normal e não possuía homogeneidade de variância. Assim, determinou-se que seria utilizado o teste não paramétrico Mann-Whitney, para estudar estatisticamente os dados, confrontando-se cada conjunto de valores simulados com os valores reais, determinados pelo professor, a fim de verificar se havia diferenças estatísticas entre cada par.

\subsection{Resultados}

Sobre os dados coletados, foi realizada uma análise estatística exploratória. Os resultados obtidos na análise são descritos a seguir.

$\mathrm{Na}$ Tabela 2, é apresentada a sumarização de algumas medidas dos dados simulados, bem como dos dados disponibilizados pelo especialista. Observando apenas os valores da tabela, pode-se verificar que o algoritmo de Similaridade dos Cossenos se destaca dentre os demais, por possuir os valores mais próximos aos reais.

Tabela 2. Sumarização de medidas para cada nível do fator estudado, além dos valores reais determinados pelo especialista.

\begin{tabular}{|c|c|c|c|c|c|c|c|}
\hline \multirow{5}{*}{$\frac{\mathscr{\sigma}}{\frac{\sigma}{\sigma}}$} & Valores & Minímo & Q1 & Mediana & Média & Q3 & Máximo \\
\hline & Similaridade dos Cossenos & 0,2425 & 0,4201 & 0,4537 & 0,4534 & 0,5145 & 0,6860 \\
\hline & Distância Euclidiana & 0,8339 & 0,8467 & 0,8503 & 0,8521 & 0,8578 & 0,8871 \\
\hline & Similaridade de Jaccard & 0,0588 & 0,1765 & 0,2059 & 0,2154 & 0,2647 & 0,4706 \\
\hline & Dice's Coefficient & 0,1111 & 0,3000 & 0,3415 & 0,3456 & 0,4186 & 0,6400 \\
\hline 参 & Especialista & 0,0000 & 0,3000 & 0,5000 & 0,4649 & 0,5000 & 1,000 \\
\hline
\end{tabular}

A realização de análise descritiva dos dados possibilitou a verificação da necessidade de utilização de um teste não paramétrico, visto que, após a análise detalhada dos dados, estes se mostraram não normais e heterogêneos. $\mathrm{O}$ teste escolhido foi Mann-Whitney, cujos resultados são apresentados na Tabela 3, para um nível de significância de 5\% $(\alpha=0,05)$.

Tabela 3. Resultados do teste Mann-Whitney, para cada função de similaridade estudada e valores reais determinados pelo especialista.

\begin{tabular}{|c|c|}
\hline Algoritmos & p-valor encontrado \\
\hline Similaridade dos Cossenos - Notas do Especialista & 0,1821 \\
\hline Distância Euclidiana - Notas do Especialista & $0,1481 \mathrm{E}-13$ \\
\hline Similaridade de Jaccard - Notas do Especilista & $0,1274 \mathrm{E}-08$ \\
\hline Dice's Coefficient - Notas do Especialista & 0,0045 \\
\hline
\end{tabular}

Buscando reafirmar as conclusões advindas da aplicação do teste não paramétrico, calculou-se o Erro Quadrático Médio (Mean Squared Error - MSE), cujos resultados encontram-se na Tabela 4. A função de Similaridade dos Cossenos apresenta 
uma taxa de erro de cerca de $3 \%$, obtendo o melhor resultado em relação às demais funções.

Tabela 4. Erro Quadrático Médio (MSE) para cada função de similaridade estudada.

\begin{tabular}{|c|c|c|}
\hline Algoritmos & Erro Quadrático Médio & Intervalo de Confiança ( $\boldsymbol{\alpha}=\mathbf{0 , 0 5})$ \\
\hline Similaridade dos Cossenos & 0,0323 & {$[0,0146 ; 0,0490]$} \\
\hline Distância Euclidiana & 0,1863 & {$[0,1318 ; 0,2408]$} \\
\hline Similaridade de Jaccard & 0,0948 & {$[0,0667 ; 0,1220]$} \\
\hline Dice's Coefficient & 0,0478 & {$[0,0307 ; 0,0648]$} \\
\hline
\end{tabular}

\subsection{Avaliação da Validação}

Em relação à validade do experimento, o fato de que o estudo foi realizado utilizando-se mensagens de fóruns com baixa atividade, devido ao curto período em que estiveram ativos, pode ser citado como uma ameaça à validação externa. Este tipo de validação está relacionado à equivalência entre as conclusões geradas pelo o estudo da amostra em relação a sua população original.

Em geral, os fóruns de discussão em ambientes de $\mathrm{EaD}$ podem gerar um volume relativamente grande de postagens, dependendo da quantidade de alunos e do tempo em que permanecem abertos. Dessa forma, os dados podem se comportar de forma diferente em ambientes com maior fluxo de postagens.

Para a validade de construção, que diz respeito às medidas utilizadas, pode-se citar a utilização de um único indicador de avaliação (palavras-chave), que corresponde a uma medida objetiva, não levando em consideração a subjetividade do docente aplicada na avaliação de discentes e a influência do fator humano. Ambos possuem papel fundamental no ambiente de avaliação real.

A validação interna corresponde a verificar se o efeito ocorrido nas variáveis dependentes foi resultado de manipulação das variáveis independentes. No contexto do experimento realizado, pode-se citar a natureza textual dos dados da amostra, que, sendo extraídos de um ambiente real e mesmo após tratamento, ainda pode conter erros.

\section{Discussão dos Resultados}

Após a análise estatística, à luz dos questionamentos previamente formulados, foram verificados indícios de que há diferença estatística entre a utilização das funções estudadas. Observando-se os resultados apresentados na Tabela 3, pode se concluir que os algoritmos de Distância Euclidiana, Similaridade de Jaccard e Dice's Coefficient apresentaram diferença estatística quando comparados com os valores reais, i.e., essas funções não apresentam resultados que representem adequadamente a população na qual o experimento está inserido.

É possível verificar que não existem diferenças significativas entre a aplicação da função de Similaridade dos Cossenos e os valores atribuídos no ambiente real por um especialista. Assim, do ponto de vista estatístico, os melhores resultados foram obtidos a partir da utilização deste algoritmo. Tal afirmação é reforçada por esta função também apresentar, as menores média e mediana para o erro. 
Desta forma, foram obtidas respostas para ambas as indagações propostas, enquanto os resultados demonstram que é possível a aplicação de funções de similaridade na verificação da participação de alunos, com resultados positivos. $\mathrm{O}$ uso desta técnica pode representar uma forma efetiva de avaliar as mensagens automaticamente, proporcionando a economia do tempo e esforços necessários para a avaliação tradicional destas mensagens realizada pelo professor.

No entanto, é importante ressaltar que, apesar da confirmação de que o uso do indicador estudado pode ser utilizado de forma satisfatória para a avaliação das mensagens dos fóruns, o uso de apenas um indicador para o problema principal da pesquisa (a avaliação da participação do aluno em cursos de EaD) não é representativo. Este fato pode ser constatado quando os valores reais são comparados com os simulados, uma vez que não é possível capturar a subjetividade existente na avaliação realizada pelo professor. O professor, da forma convencional, é capaz de analisar fatores como cópias de textos existentes na web, apresentação de vídeos e imagens relevantes ao tema, conversas individuais com o tutor, dentre outros fatores. Neste contexto, outros indicadores também podem ser utilizados, como os indicadores sociais.

\section{Considerações Finais}

O estudo-piloto confirmou que é possível empregar funções de similaridade no contexto da avaliação da participação de alunos em cursos à distância. Em se tratando do estudo destas funções, o algoritmo da Similaridade dos Cossenos apresentou resultados superiores aos demais e confirmou os benefícios trazidos pelo emprego de técnicas da IA para o tipo de problema abordado. Portanto, pôde-se verificar a viabilidade de implantar técnicas de IA, como Raciocínio baseado em Casos/Regras, usando medidas de similaridade.

Por se tratar de um estudo inicial, apenas um indicador de avaliação da participação foi considerado. E, embora o erro encontrado tenha sido relativamente pequeno, ainda existem limitações para sua aplicação de forma isolada, por ser um indicador objetivo. Durante a avaliação de alunos, não apenas em cursos à distância, mas também presencial, há forte presença da subjetividade. Estudos mais aprofundados sobre como é realizado o processo de avaliação pelos professores desta modalidade de ensino já estão em andamento. Pretende-se, com isso, verificar a viabilidade de empregar novos indicadores, associados ao uso de medidas de similaridade, como apresentado neste trabalho. Como proposta de aprimoramento dos resultados da pesquisa, sugere-se realizar o estudo de forma a generalizar as conclusões, adicionando dados de cursos de diferentes áreas e com diferentes níveis de atividade.

\section{Referências}

Andresen, M. (2009) Asynchronous discussion forums: success factors, outcomes, assessments, and limitations. Em: Educational Technology \& Society, p. 249-257. Volume 12, Edição 1.

Blackboard Inc. (1997) Blackboard Learning System. Sistema de gestão da aprendizagem. 
Calvani, A., Fini, A, Molino, M. e Ranieri, M. (2009) Visualizing and monitoring effective interactions in online collaborative groups. Em: British Journal of Educational Technology, p. 213-226. Volume 41, Edição 2.

Dougiamas, M. (2001) MOODLE - Modular Object-Oriented Dynamic Learning Environment. Sistema de gestão da aprendizagem.

Elia, M. e Chamovitz, I. (2009) CQMsg - Classificador e Qualificador de Mensagens: um instrumento para apoio à avaliação de fóruns temáticos. Em: Anais do XX SBIE Simpósio Brasileiro de Informática na Educação. Santa Catarina, Brasil.

Erlin, Yusof, N. e Rahman, A. (2009) Students' interactions in online asynchronous discussion forum: A social network analysis. Em: International Conference on Education Technology and Computer, p. 25-29.

FOSS - Free and Open-source Software (2012). Eclipse, 4.2 Juno. Software development environment.

Hrastinski, S. (2008) What is online learner participation? A Literature Review. Em: Computers \& Education, p. 1755-1765. Volume 51, Edição 4.

Ihaka, R. e Gentleman, R. (1993). R Statistics, versão 2.15.1. Software environment for statistical computing and graphics.

Júnior, R. L. O., Esmin, A. A. A., Coelho, T. A., Araujo, D. L., Alosno, L. e Giroto, R. (2011) Uma ferramenta de monitoramento automático de mensagens de fóruns em ambientes virtuais de aprendizagem. Em: Anais do XXII SBIE - Simpósio Brasileiro de Informática na Educação. Sergipe, Brasil.

Júnior, R. L. O. e Esmin, A. A. A. (2012) Monitoramento automático de mensagens de fóruns de discussão usando técnica de classificação de texto semi-supervisionado. Em: Anais do XXIII SBIE - Simpósio Brasileiro de Informática na Educação. Rio de Janeiro, Brasil.

Leite, E. A. M., Sales, G. L., Sousa, L. L. R. e Joye, C. R. (2011) Avaliação assistida, feedbacks e questionários do moodle. Em: Anais do XXII SBIE - Simpósio Brasileiro de Informática na Educação. Sergipe, Brasil.

Lin, F., Hsieh, L. e Chuang, F. (2009) Discovering genres of online discussion threads via text mining. Em: Computers \& Education, p. 481-495. Volume 52, Edição 2.

Romero, C., López, M., Luna, J. e Ventura, S. (2013) Predicting students' final performance from participation in on-line discussion forums. Em: Computers and Education, p. 458-472. Volume 68.

Tan, P., Steinbach, M. e Kumar, V. (2005) Introduction to Data Mining. AddisonWesley Longman Publishing Co., 1a Edição. 